\title{
Uncovering Frustrations
}

A Qualitative Needs Assessment of Academic General Internists as Geriatric Care Providers and Teachers

Craig E. Tanner, MD, ${ }^{7}$ Elizabeth Eckstrom, MD, MPH, ${ }^{2}$ Sima S. Desai, MD, ${ }^{3}$

Carol L. Joseph, MD, ${ }^{7}$ Marnie R. Ririe, MD, ${ }^{2}$ Judith L. Bowen, MD ${ }^{3}$

'Oregon Health \& Science Univ./Portland VA Medical Center, Portland, OR, USA; ${ }^{2}$ Legacy Health System, Portland, OR, USA;

${ }^{3}$ Oregon Health \& Science University, Portland, OR, USA.

BACKGROUND: General internists commonly provide medical care for older adults and geriatric education to trainees, but lack the necessary knowledge and skills to fulfill these tasks.

OBJECTIVE: Assess the geriatric training needs of academic general internists in 3 hospital systems in Portland, OR.

DESIGN: Ten focus groups and 1 semi-structured interview. Interview transcripts were analyzed using thematic analysis, a well-recognized qualitative technique.

PARTICIPANTS: A convenience sample of 22 academic general internists and 8 geriatricians from 3 different teaching hospitals.

MEASUREMENTS: We elicited stories of frustration and success in caring for elderly patients and in teaching about their care. We asked geriatricians to recount their experiences as consultants to general internists and to comment on the training of Internists in geriatrics.

RESULTS: In addition to deficits in their medical knowledge and skills, our Internists reported frustration with the process of delivering care to older adults. In particular, they felt ill prepared to guide care transitions for patients, use multidisciplinary teams effectively, and were frustrated with health care system issues. Additionally, general internists' approach to medical care, which largely relies on the medical mod$\mathrm{el}$, is different from that of geriatricians, which focuses more on social and functional issues.

CONCLUSIONS: Although our findings may not be broadly representative, improving our general internists' abilities to care for the elderly and to teach learners how to do the same should address deficits in medical knowledge and skills, barriers to the processes of delivering care, and philosophical approaches to care. Prioritizing and quantifying these needs and measuring the effectiveness of curricula to address them are areas for future research.

KEY WORDS: geriatrics; general internal medicine; medical education; faculty development; qualitative methods; curriculum development. DOI: $10.1111 / \mathrm{j} .1525-1497.2005 .00281 . \mathrm{X}$

J GEN INTERN MED 2006; 21:51-55.

$\mathrm{T}$ he shortage of geriatricians and the aging of the U.S. population have been well described for at least 2 decades. ${ }^{1}$ As a result of this shortage, general internists now provide a substantial portion of medical care for the elderly and, in academic settings, are frequently responsible for teaching geriatrics to students and residents. Despite this fact, many general internal medicine faculty lack the necessary knowledge and skills to prepare future physicians for their roles in caring for the aging population. ${ }^{2}$ Multiple initiatives are underway to address these gaps in practice and teaching skills. ${ }^{3,4}$ Some teaching programs have developed sophisticated geriat-

None of the authors has any conflicts of interest to disclose.

Address correspondence to Dr. Bowen: Oregon Health and Science University, 3181 SW Sam Jackson Park Rd, Mail Code LA75, Portland, OR (e-mail: bowenj@ohsu.edu). rics curricula and faculty development programs that address deficits in general internists' knowledge and skills. ${ }^{3}$ While these efforts are necessary, are they sufficient?

As part of the Society of General Internal Medicine Collaborative Centers for Research and Education in the Care of Older Adults, a Hartford Foundation-sponsored initiative, we developed a geriatric-focused faculty development program for academic general internists. This paper reports the results of the needs assessment we conducted to inform the development of our curriculum. Previously published needs assessments in this area have mainly focused on geriatric medical knowledge. ${ }^{5,6}$ While this is clearly an important part of any faculty development program, our own experiences suggested that frustrations in caring for older adults and educating trainees about their care are more complex than simply addressing gaps in medical knowledge. A qualitative needs assessment involving internal medicine and family medicine residents identified the "health care system" and "societal and cultural issues" as important domains for residents in caring for older adults. ${ }^{7}$ Our objective was 2 -fold: first, to gain a deeper and broader understanding of the frustrations and training needs of our general internists in caring for and teaching about the elderly and their care, and second, to elicit the perspectives of geriatric experts on the care provided by internists.

\section{METHODS}

\section{Sample and Data Collection}

Letters were mailed to all academic general internists from 3 institutions (a community-based teaching hospital, a university hospital, and a Veterans Affairs hospital) and academic geriatricians from 2 of the 3 institutions (the community-based teaching hospital and the VA hospital) in Portland, OR, explaining the project and inviting their participation in focus groups or individual interviews. No geriatricians were employed by the university hospital at the time of the data collection. A total of 53 general internists and 11 geriatricians were invited to participate in the interviews. Participation was voluntary. We chose focus groups as our primary method to trigger participants' recall of their experiences in caring for older adults when listening to others' stories. We did not try to reach consensus about any subject within a focus group. Rather, we desired the breadth of experiences. When we were unable to schedule a participant for a focus group because of schedule conflicts, willing participants were offered an individual semi-structured interview.

Two members of the research team familiar with qualitative methods and focus group techniques developed the ques-

Manuscript received December 10, 2004

Initial editorial decision March 14, 2005

Final acceptance August 30, 2005 
tions used in the focus groups and semi-structured interviews. The questions used were intended to elicit stories from participants as clinicians and teachers caring for older adults. Such storytelling has been shown to help uncover subconscious needs. ${ }^{8}$ Furthermore, narratives can offer an insight into complex meanings and relationships between concepts that are not overtly stated. ${ }^{9}$

A trained research assistant conducted the focus groups and interviews with the university-based and VA-based participants. A trained general medicine fellow conducted the focus groups with the community hospital-based participants. General internists were asked to recall specific successful as well as frustrating experiences in caring for elderly patients, and specific successes and difficulties that they had encountered in teaching students and residents about the care of older adults. Geriatricians were asked to recall both positive and negative situations where they served as consultants for general internists, their perceptions of general internists' skills in caring for older adults, and aspects of geriatrics they found particularly satisfying. They were also asked to comment on the current training of internal medicine residents in the care of older adults. The questions were intentionally broad and open-ended, and the interviewers were encouraged to pursue in more detail any important lines of discussion that emerged during the sessions. No analysis of data was undertaken during or between the focus group sessions, and neither interviewer was initially intended to be involved in data analysis. After data collection was completed, the general medicine fellow who had interviewed the community hospital-based participants joined the research team and was involved in all subsequent analyses.

Separate focus groups were conducted for general internists whose primary role was outpatient clinical teaching, general internists serving as teaching hospitalists, and geriatricians. All focus groups and interviews were recorded and transcribed verbatim, with identifying data removed prior to analysis. The institutional review boards of all the institutions involved approved the project, and written informed consent was obtained from all participants.

\section{Data Analysis}

We used the method of thematic analysis and text coding described by Ryan and Bernard ${ }^{10}$ to examine the interview transcripts and to identify emerging themes. The research team included 2 outpatient general internists (E.E., J.B.) experienced in qualitative analysis, 1 hospitalist (S.D.) experienced in qualitative analysis, 1 geriatrician (C.J.), and 2 general medicine fellows (C.T., M.R.). Transcripts were organized into sets by participant subgroup: outpatient Internists, hospitalists, and geriatricians. Each team of 2 investigators initially examined 1 set of transcripts and generated a preliminary coding scheme. The entire research team then met and discussed the coding schemes until a consensus was obtained on the coding categories. Each team then analyzed a second set of transcripts with the new scheme. The entire group met again to review the coding with this scheme until a consensus was reached on a final master coding scheme. The research teams then re-coded their sets of transcripts using the final coding scheme. Each set of transcripts was independently coded by at least 4 investigators. One investigator coded all of the transcripts (C.T.). Differences in coding results using the final scheme were resolved through discussion until a consensus was achieved. Consensus was achieved in all cases. Because the unit of analysis was highly variable, ranging from brief phrases to paragraphs, and paragraphs were often coded with more than 1 interrelated theme, no frequency counts were made for the codes and, as a result, interrater reliability was not calculated.

\section{RESULTS}

A total of 30 physicians participated in the project (11 outpatient Internists, 11 hospitalists, and 8 geriatricians). This sample represented $42 \%$ of the general internists who were invited to participate and $73 \%$ of the invited geriatricians. Only 1 participant (an outpatient Internist) was unable to participate in any of the focus groups and was therefore interviewed individually. When the research team reviewed the transcript from this individual interview, no substantive differences were seen when compared with focus group transcripts. This transcript, therefore, was collapsed into the focus group transcripts and analyzed along with them. Ten focus groups (ranging in size from 2 to 5 participants) were conducted with the remaining 29 participants.

The coding scheme is shown in Table 1. Three major themes were identified. Each theme is elaborated below, and representative quotes are included. Some quotes have been edited slightly for readability. Differences between outpatient General Internists, hospitalists, and geriatricians are be noted.

\section{Geriatric Medical Knowledge/Skills}

This theme showed the most agreement among the 3 groups, indicating a strong consensus on the General Internists' perceived knowledge and skill gaps in caring for older adults. The most commonly mentioned knowledge deficits were in the areas of medication prescribing (polypharmacy), neurologic and behavioral problems (dementia, delirium, cognitive dysfunction, depression), urinary incontinence, and falls. Additionally, geriatricians and outpatient Internists discussed knowledge gaps in appropriate health screening in the elderly, and hospitalists discussed gaps in the area of ethical issues, such as the capacity to make treatment decisions.

Participants described clinical skills deficits including cognitive and functional assessments, assessment of gait and falls, and evaluating decision-making capacity. They also mentioned lack of skills in performing medication and social assessments. Knowledge and skill deficits were closely linked. A lack of medical knowledge or skills expertise was the most frequently mentioned barrier to teaching residents about caring for older adults effectively:

Inpatient internist: “ . . . the problem is that most [general internists] have had no formal training in geriatrics. So I think that you are talking to a group of physicians for whom geriatrics was probably not even a word that we even really thought about until the last 5 years. So we would probably not really be the resources for residents..."

Inpatient internist: "She had incontinence in the night, got confused, stood up and because the urine had ended up on the floor, she slipped, she fell, she hit her head ... and the next morning we say we ought to talk about how we can help this woman with her urinary incontinence and I stood there and stared at [the residents]. That is a bummer." 


\section{Table 1. Coding Scheme}

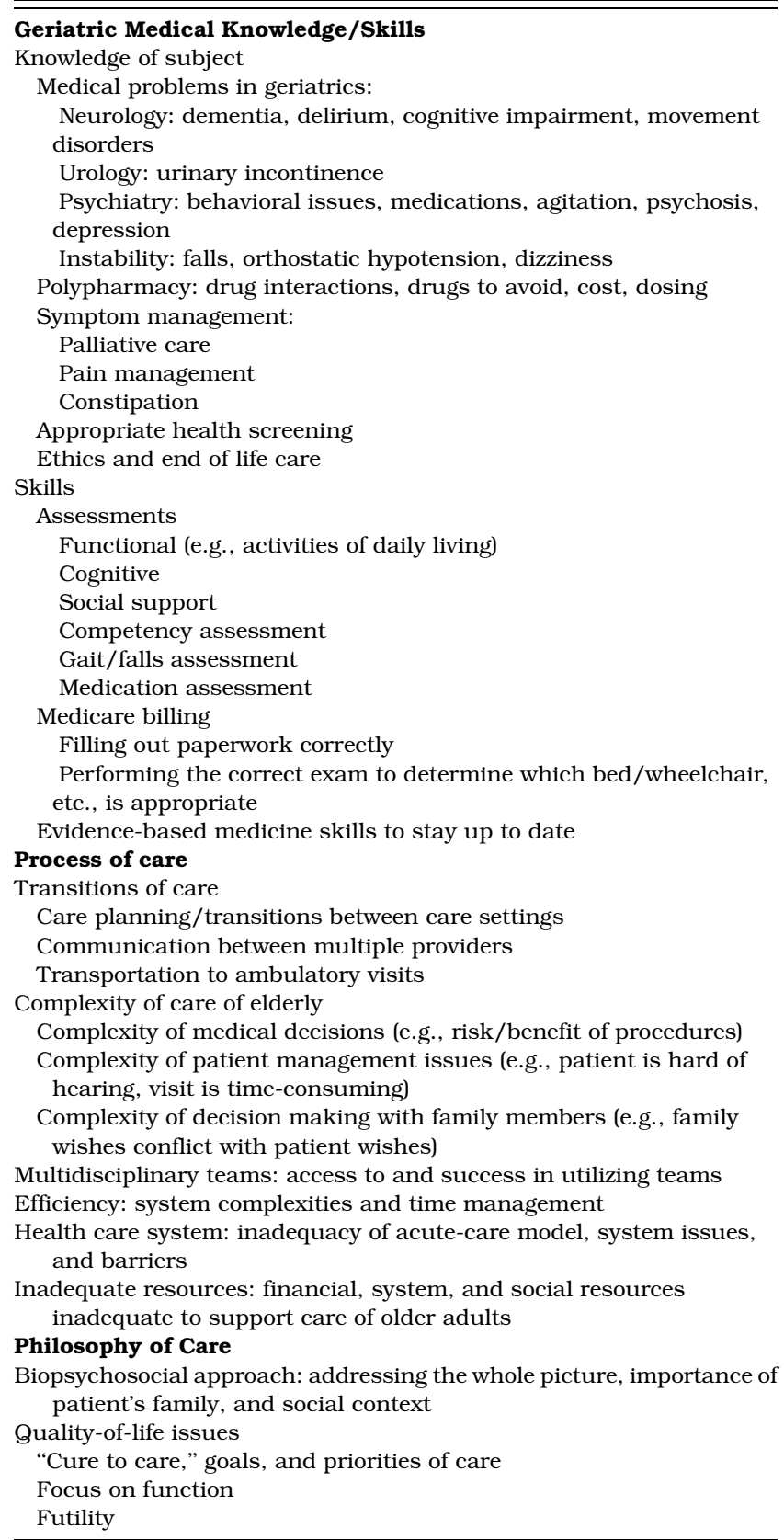

Outpatient internist: "Here's the evidence-based box of items that we need to address. We know it for all these diseases but we don't know it for functional decline. What we don't know we can't teach ... [Residents] are basically getting precepted by people who don't know how to do it."

\section{Process of Care}

Both hospitalist and outpatient general internists expressed concerns about their abilities to manage care processes for older adults. Geriatricians did not identify this as a source of frustration for themselves, but did recount situations in which they had observed Internists' shortcomings in this area. The subthemes of care transitions and care planning, time constraints, the use of multidisciplinary teams, and the availabil- ity of resources to care effectively for older adults are included here. Some of the frustrations described under this heading reside in the health care system, some in the physician's ability to navigate the system, and others in the complex medical and social situations presented by older patients themselves.

Navigating patients' care transitions to or from the hospital and other care facilities was a common source of frustration for General Internists. Inpatient Internists focused on discharge-planning difficulties for patients transitioning to care facilities, while outpatient Internists discussed challenges regarding decisions about bringing in patients from care facilities for evaluation.

Inpatient Internist: "One frustration I've had with caring for geriatric patients is finding the right place for discharge." Geriatrician: "We still have hospitalists who think when they send people to assisted living that they are going to a skilled nursing facility."

Outpatient Internist: (discussing a patient in a care facility) "Are they in a setting that has nurses and no physicians but may have urinalysis capabilities and medication dispensing and physical therapy available? Sometimes we recommend patients come in to the emergency room to be evaluated when they probably could have most of their evaluation done at a facility but we just don't know what type of services are there."

The geriatricians and outpatient Internists frequently mentioned the inadequacy of the current system's acute care, short visit design for seeing geriatric patients. Inpatient Internists did not discuss these concerns.

Geriatrician: “ . . . internists are not able to address all of the issues because of time, not because they don't know, they just don't have time ... I just have time to work the things out with the family members and the patient."

Outpatient Internist: "[We] find out what the symptom is ... then treat that. We don't talk about how far can you walk and how far do you want to walk. I think those things are important. But sometimes we don't want to go there because we don't have the time to open up that box."

Outpatient internist: "Geriatric patients have not just medical issues, but functional, social, and cognitive issues. We don't get any more time to see them than to see the 40 -year-old with high blood pressure."

All 3 groups recognized the importance of multidisciplinary teams in delivering quality care to the elderly, but general internists in both the inpatient and outpatient settings had variable access to or success with using such teams:

Outpatient internist: "in our clinic there's no coordinated team effort ... it's the health care model we work in that doesn't pay for a multidisciplinary team."

Inpatient internist: (when describing research on team approaches to the treatment of delirium) "It needs to be a multidisciplinary team approach to the patient with delirium and patients with that approach do better . . . but it doesn't work in the real world." Geriatrician: “. . . we have more contact with social workers, pharmacists, therapists of many different fields ... we all learn from each other in more of an integrated team setting than internists do ..."

\section{Philosophy of Care}

References to the philosophy of care appeared in all the transcripts. The subthemes under this heading included the importance of addressing patients' needs within the context of their life goals and social situations, the importance of involving patients' families in care planning and decisions, the shift 
in focus from "cure" to "care" in the elderly, and the need to focus on quality of life and functional status.

Geriatricians described their approach to patients as different from General Internists:

Geriatrician: "The internist approach is more a pathophysiological approach. But the geriatrician approach is a more social function approach."

Geriatrician: "[W]e learn to deal with the family too, so it's not just the patient alone. We learn to handle the family too."

When asked to describe successful encounters with elderly patients, general internists nearly always recounted a situation in which they had discussed the patient's wishes and goals, and coordinated closely with the patient's family.

Inpatient internist: "She's got a family that wants lots of information and doesn't know what the right decision is and I remember ... just developing a relationship with the family and the patient at that time was really rewarding... I got all kinds of feedback from them that was positive ... so that feels like a success."

Inpatient internist: "The family's just at their wits end and you really helped them cope with a plan that feels good to them to deal with their loved one. Those are good experiences when they go well."

Both geriatricians and outpatient Internists described the lack of a global, functional approach as a shortcoming of general internists in their care of older adults:

Geriatrician: "You watch [medicine residents] do a history and physical ... they'll tell you the patient has shortness of breath but they don't have a clue as to where the person lives, who takes care of them."

Outpatient internist: "We ask about how your breathing is ... we don't ask things like what aren't you able to do because of your shortness of breath ... we don't go to function."

Although all 3 groups spoke of the shift in focus from "cure" to "care," the general internists in both settings often described this issue as a source of frustration:

Inpatient internist: "We have an 84-year-old woman with interstitial lung disease, which is probably end stage, coming in with an acute exacerbation of her underlying [disease] ... The team clearly got frustrated on day six and thought she should be in comfort care."

Outpatient internist: "One of the things that is difficult is trying to figure out where that line is, as far as how much diagnostic work up to do on a patient ... it's tough for me to figure out where that line is, let alone convince the patient where the line is."

Geriatrician: "[Internists] need to understand that maybe cure is not possible anymore, but we can improve quality of life."

Medical futility and ambivalence about continuing aggressive care in the face of such futility was also raised. This theme appeared only in the hospitalist transcripts.

Inpatient internist: "[A patient who] had heart failure with an $\mathrm{EF}$ [ejection fraction] of $10 \%$ and had suffered multiple strokes. He came in with a heart failure exacerbation, was intubated, put on pressors et cetera, and when I talked to the family about his longterm prognosis and futility, they would only talk about their belief in miracles. And I found that really frustrating."

\section{DISCUSSION}

We sought to identify the needs of Internists caring for geriatric patients and the influence of these needs on their perceived teaching abilities. Our goal was to inform the development of a geriatrics curriculum for General Internist clinician-educators. The use of focus groups allowed us to identify gaps in medical knowledge and skills that our Internists perceived, as well as other sources of frustration they face in caring for the elderly and in teaching residents about caring for this population.

The reported knowledge and skill gaps we identified are consistent with those found in previously reported needs assessments: "geriatric syndromes" (incontinence, falls, delirium, dementia, cognitive dysfunction, and polypharmacy), health screening, and ethical issues. ${ }^{5,6}$ Lack of expertise in these domains was the most frequently identified barrier to teaching residents effectively about the care of older adults. Our study also identified 2 additional domains as sources of frustration in caring for older adults: process of care issues and philosophy of care. The general internists in our study expressed a great deal of frustration with time constraints in caring for geriatric patients, facilitating transitions of care, and working effectively in multidisciplinary teams. The geriatricians and general internists alike recognized the dominance of the "medical" model and lack of a social-functional approach in general internists' care of the elderly.

What are the implications of our results for curriculum development? The study Internists and geriatricians generally agreed on the medical topics that should be included in our faculty development program. The shifting demographics of the adult population and shortage of geriatricians require us to address these gaps and improve our General Internists' confidence in delivering quality health care to the aging population and in teaching others to do the same. More difficult to answer are questions about the level of competence or expertise we should expect from Internists' care delivery and educational efforts.

In our experience, issues related to processes of care are rarely addressed in internal medicine curricula. Perhaps the idiosyncratic nature of these issues in varied health care systems makes it impractical to address them at a curricular level. Frustrations with processes of care are likely present in nearly all health systems and care settings, and it is possible that those we identified are particular to the systems in which our study participants work. This does not seem to be the case in our data. Despite working in 3 fairly disparate health care settings (VA, public university, and private community), we found that the frustrations with care processes described in the focus groups were quite similar.

Alternatively, physicians may rarely identify the process of care issues as important targets of educational interventions. Some support for this notion comes from a previously reported needs assessment by Williams and Connelly, ${ }^{6}$ which included reference to topics such as "family and social issues" and "nursing home decisions." In this study, physicians were asked to choose the topics that would be important enough to attract them to a continuing medical education program. The topics of "family and social issues" and "nursing home decisions" were chosen less frequently than medical topics.

Our results relate only to the process and system issues in our own settings; however, such issues are increasingly being identified as important to medical education. For example, The Accreditation Council for Graduate Medical Education outcomes project has recognized the importance of systems issues in graduate medical education and has defined "systems-based practice" as 1 of its core competencies. ${ }^{11}$ Incorporating such content into faculty development programs in geriatrics might serve to reduce General Internists' frustrations, and also aid our residency program in addressing this competency. 
In addition to medical knowledge, skills, and process-related frustrations, the geriatricians and Internists in our study also identified differences in philosophical approaches to care of the elderly. It is interesting that the study Internists describe great difficulty in utilizing a more global, social-functional approach to their care of elderly patients while the geriatricians embrace it. Perhaps during training, Internists' exposure to geriatrician role models and their approaches in caring for older adults is very limited, with the medical model predominating. Alternatively, the specialty of geriatrics may select for physicians who have an interest in or are skilled in a socialfunctional approach. Finally, the specialized training and experience provided during geriatric fellowships may be critical in developing this perspective.

Process- or system-related factors may also shape one's philosophical approach to caring for the elderly. For example, geriatricians in our sample appear to have more time to address social and functional issues. Clinic-based Internists express frustrations over time-limited visits and tend to put off discussing functional and social issues. It is difficult to know whether these differences represent an effect of training and expertise, actual appointment structure differences, or different philosophical approaches to care. Future research could address which of these possibilities accounts for the differences observed.

Perceived lack of medical knowledge and skills was frequently cited by our participants as a barrier to teaching effectively about the care of older adults, while difficulties with processes of care or differences in philosophies of care were not. The reasons for this are not clear, although the acknowledged tendency of our Internists to focus on medical more than social and functional issues would be consistent with the view that medical knowledge deficits are the most prominent barriers to teaching.

Our study has several limitations. First, our sample size is limited to general internists and geriatricians with teaching roles in 1 city ( 3 institutions). It is possible that additional themes would emerge in other academic settings, or that the perspectives described may be unique to those who volunteered to participate for this project. The fact that our results in the domains of knowledge and skills closely resemble those obtained by others ${ }^{5,6}$ suggests that at least some of what we describe is common to other settings and systems. We did involve physicians from 3 diverse health care systems in 1 city to obtain as broad a range of perspectives as possible.

Second, our interview questions were different for general internists and geriatricians, limiting our ability to compare results directly across specialties. Our goal for including geriatricians was to incorporate their perspectives as consultants, i.e., specialists, whose role is to help meet the needs of general internists in caring for the elderly. Subsequent research using parallel questions could provide a more direct comparison.

Third, our participants disproportionately represented their professional groups. While 8 of 11 (73\%) geriatricians agreed to participate, only 22 of $52(42 \%)$ general internists participated. It was easier to schedule focus groups with a small number of geriatricians, as they work closely together in discreet geriatric care delivery sites. It is possible that the General Internists' frustrations are less representative of the group as a whole, even though the number of pages of transcripts gathered from general internists was larger.

Fourth, the depth and richness of data obtained using qualitative methods allowed us to gain an insight into issues beyond those previously identified in the literature. Nonetheless, these methods do not allow us to generalize or quantify our results. They could, however, form the basis for future quantitative assessment of the identified needs and gaps in larger populations of academic physicians. This may be particularly true for the areas of care processes and philosophies, as quantitative measurements in larger populations could elucidate similarities and differences within and between systems.

Our qualitative assessment of the perceived geriatric training needs of academic general internists from 3 different settings in the same city identified multiple gaps in skills and knowledge. We also uncovered 2 new domains not described in the literature on general internists caring for older adults: improving process knowledge and skills for delivering care to older adults and appreciating the geriatrician's philosophy of care. We believe that these areas impact faculty knowledge and skills, confidence in teaching, learner satisfaction with training in geriatrics, and patient outcomes and satisfaction, and are prime areas for further study.

The John A. Hartford Foundation provided support for this project to the Society of General Internal Medicine through a grant (Grant number 2002-0013).

\section{REFERENCES}

1. Kane R, Solomon D, Beck J, Keeler E, Kane R. The future need for geriatric manpower in the United States. N Engl J Med. 1980;302:132732 .

2. Rubin C, Stieglitz H, Vicioso B, Kirk L. Development of geriatrics-oriented faculty in general internal medicine. Ann Intern Med. 2003;139:615-20.

3. Swagerty D, Walling A, Studenski s. Preliminary report from the Kansas Hartford Geriatrics Project: a model of community university collaboration in geriatric medicine faculty development. J Am Geriatr Soc. 2000;48:1513-8.

4. Hazzard W, Woolard N, Regenstreif $\mathbf{D}$. Integrating geriatrics into the subspecialties of internal medicine: the Hartford Foundation/American Geriatrics Society/Wake Forest University Bowman Gray School of Medicine Initiative. J Am Geriatr Soc. 1997;45:638-40.

5. Robinson B, Barry P, Renick N, Bergen M, Stratos G. Physician confidence and interest in learning more about common geriatric topics: a needs assessment. J Am Geriatr Soc. 2001;49:963-7.

6. Williams M, Connolly N. What practicing physicians in North Carolina rate as their most challenging geriatric medicine concerns. J Am Geriatr Soc. 1990;38:1230-4.

7. Chodosh J, Tulsky A, Naumberg E, et al. What residents want to know about geriatrics: an approach to curriculum development. Gerontol Geriatr Educ. 1999;20:19-35.

8. Gustafson DH, Taylor JO, Thompason S, Chesney P. Assessing the needs of breast cancer patients and their families. Qual Manage Healthcare. 1993;2:6-17

9. Patton MQ. Conceptual issues in qualitative inquiry. In: Patton MQ, ed. Qualitative Research \& Evaluation Methods. 3rd edn. Sage; 2002:115-8.

10. Ryan G, Bernard HR. Data management and analysis methods. In: Denzin NK, Lincoln YS, eds. Handbook of Qualitative Research. 2nd edn. Thousand Oaks, CA: Sage; 2000:769-802.

11. ACGME. Outcomes project, 2003. Chicago, IL: Accreditation Council for Graduate Medical Education. Available at: www.acgme.org/Outcome. Accessed November 2, 2004. 